\title{
INFLUENCIA DE LA SALINIDAD SOBRE LAS POBLACIONES ALGALES DE TRES LAGUNAS LITORALES (Albuferas de Adra, Almería)
}

\author{
Pedro M. Sánchez Castillo \\ Departamento de Botánica, Facultad de Ciencias, Universidad de Granada, 18001 Granada.
}

Palabras clave: Fitoplankton, coastal lagoons, Albuferas of Adra (Almería, Spain).

\begin{abstract}
SALINITY INFLUENCE ON ALGAL POPULATIONS FROM THREE COASTAL LAGOONS

(Albuferas de Adra, Almería)
\end{abstract}

The «Albuferas» from Adra (Almería,Spain)constitute a set of three sea-side lagoons with a parallel disposition to the coast line. In this paper we relate their chloride concentrations to the phytoplankton taxonomic composition. From the data obtained it is evident that in the two inland ones, with chloride values, over the annual cycle, lesser than $2.5 \mathrm{gr} / 1$, Merismopedia punctata, Coelosphaerium minutissimum, Peridinium sp. and Bacillaria paradoxa are the most frequent species. The litoral lagoon, reaching chloride values up to $13 \mathrm{gr} /$, shows a phytoplankton clearly dominated by halofic species like Gomphosphaeria uponina. Achnanthes brevipes, Diploneis didyma, Mastogloia braunii and Tetraselmis subcordiformis.

\section{INTRODUCCION}

La franja costera de la provincia de Almería se encuentra salpicada por gran cantidad de lagunas litorales (Fig. 1A) de distinta procedencia; así, por ejemplo, en la desembocadura de la mayor parte de los ríos, todos ellos de carácter torrencial, existen masas de agua que surgen de la mezcla de parte de su caudal subterráneo con el agua del litoral, mientras que en otros casos están formadas fundamentalmente por el agua que aflora de los manantiales freáticos, ambos casos están representados en las lagunas de las albuferas de Adra.

Las Albuferas de Adra están formadas por tres lagunas (Fig. 1B) de disposición paralela a la línea de costa y de distinto origen, así mientras que las albuferas Honda y Nueva se pudieron haber formado mediante el arrastre de los aluviones del río Adra por los fuertes vientos de poniente reinantes en la zona, la albufera Litoral se está formando por el hundimiento progresivo de un labio de falla (Jiménez, com. pers.).

Limnética 3: 47-53 (1987)

(C) Asociación Española de Limnología, Madrid. Spain
Al haberse convertido toda la zona circundante en unos fértiles campos de cultivo, las albuferas han ido quedando totalmente rodeadas por los mismos, e incluso invadidas, sirviendo de zonas de acumulación de desechos. Por ello, desde los años cincuenta hasta nuestros días, las albuferas han sufrido un gravísimo proceso de colmatación, colmatación que al ser realizada con los restos de los cultivos va eutrofizando el agua progresivamente año tras año.

Las albuferas de Adra constituyen la zona húmeda natural de mayor importancia de todo el litoral almeriense, no sólo por su gran volumen de agua, sino por estar formada por tres lagunas de distintas características. En este trabajo damos a conocer los primeros datos sobre el estudio de su fitoplankton, del cual sólo ofrecemos su composición taxonómica y su distribución en las tres lagunas, estableciendo algunas relaciones entre la ficoflora de las lagunas y su contenido en cloruros. Del mismo modo se dan algunos datos sobre la química de sus aguas. 


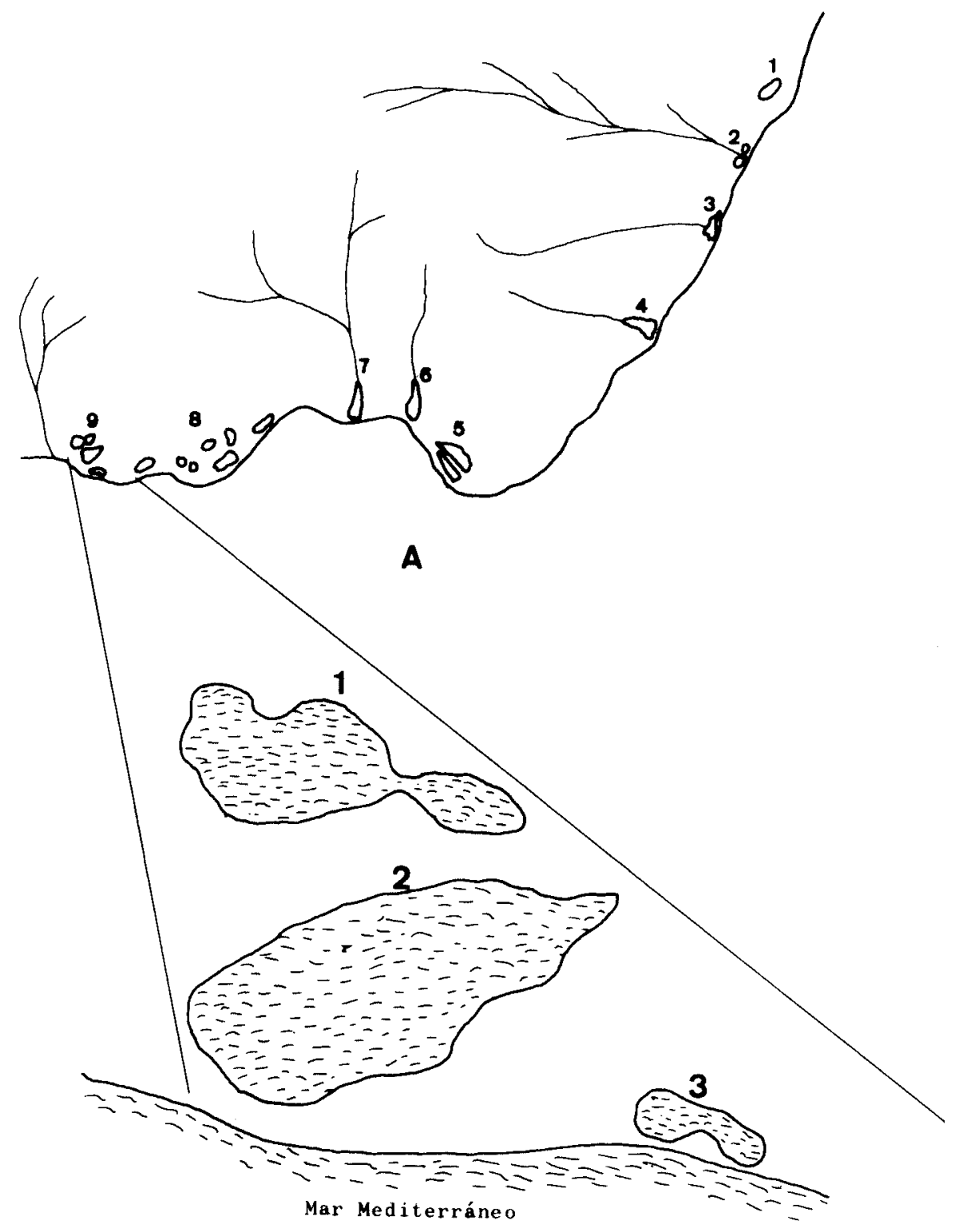

B

Figura 1.- A) Lagunas costeras del litoral almeriense.

1, San Juan de los Terreros; 2, Río Almanzora; 3, Río Antas; 4, Río Aguas; 5, Cabo de Gata; 6, Rambla Morales; 7, Río Andarax; 8. Conjunto lagunar de Punta del Sabinar; 9, Albuferas de Adra.

B) Situación de las Albuferas de Adra.

1, Albufera Honda; 2, Albufera Nueva; 3, Albufera Litoral.

A) Coastal lagoons from Almería litoral.

1, San Juan de los Terrenos; 2, Río Almanzora; 3, Río Antas; 4, Río Aguas; 5, Cabo de Gata; 6, Rambla Morales; 7, Río Andarax;

8, Lagoons complex of Punta del Sabinar; 9, Albuferas of Adra.

b) Lagoons of Adra.

1, Albufera Honda; 2, Albufera Nueva; 3, Albufera Litoral. 


\section{MATERIAL Y METODOS}

Las muestras se tomaron en un punto fijo de la zona litoral de cada una de las tres lagunas; las de fitoplancton fueron obtenidas con red de Nytal de $10 \mathrm{u}$ de tamaño de poro. Aquellas otras destinadas a la realización de los análisis químicos se conservaron con cloroformo y en frío hasta su análisis. Para la medida de la alcalinidad se utilizó el método volumétrico expuesto en Golterman \& Clymo (1969); para el fósforo el método espectrofotométrico de Murphy \& Riley (1962); y para los cloruros el de Volhard (in Golterman \& Clymo, loc. cit.). Los análisis se llevaron a cabo en agua sin filtrar, por lo que las medidas de fósforo han de considerarse, al menos en parte, como fósforo orgánico.

La periodicidad de los muestreos fue mensual durante el periodo de noviembre de 1982 a diciembre de 1983.

\section{RESULTADOS}

\section{1) APORTE HIDRICO}

El aporte hídrico, como en la mayoría de las lagunas costeras, está claramente influenciado por el agua marina, observándose un claro gradiente de salinidad entre las dos albuferas interiores y la litoral, y en menor medida entre las dos interiores: Honda y Nueva. Se puede considerar que los principales factores reguladores de la salinidad son: aporte freático, evaporación y aporte marino, haciéndose los dos últimos más patentes durante el verano. Pensamos que entre las albuferas Honda y Nueva se debe producir un importante intercambio, de la primera a la segunda durante el invierno y en sentido inverso durante el verano. La albufera Litoral, debido a su mayor distancia de las dos interiores, creemos que no debe tener comunicación con ellas, mientras que entre ésta y el mar, la comunicación es directa, debido a su gran proximidad (no más de 20 metros), lo que condiciona que durante los días de fuerte oleaje exista un aporte directo de agua marina al interior de esta albufera.

\section{2) PARAMETROS QUIMICOS}

Tanto la reserva alcalina como el $\mathrm{pH}$ se mantienen en torno a unos límites bastante constantes a lo largo de todo el ciclo anual; el pH varía, en las tres lagunas, entre valores de 7 y 8.5 , mientras que la alcalinidad oscila entre 5-10 meq/l en la albufera Honda, entre 3.3 y 9.6 en la Nueva, mientras que en la albufera Litoral se obtienen los valores más bajos: 2.1-6.3 meq $/ 1$.

La situación de estas tres lagunas respecto a la costa, así como sus posibles intercambios hídricos, antes comentados, nos hacen pensar en la existencia de un marcado gradiente en su concentración de cloruros. Efectivamente, en base a los datos representados en la figura 2, se pueden establecer dos grupos de lagunas, uno formado por la albufera Litoral y otro por las dos albuferas interiores: Honda y Nueva. Así, la albufera Litoral, además de estar directamente influenciada por el agua marina, sufre un intenso proceso de evaporación durante el verano, por lo que llega a alcanzar valores de hasta $13 \mathrm{gr} \mathrm{Cl} / 1$, comenzando a disminuir a principios del otoño con las primeras precipitaciones. Sin embargo, las dos lagunas interiores, presentan una muy marcada regulación de la salinidad, debido fundamentalmente al aporte de agua dulce que reciben de su manantial freático, de forma que no superan el valor de $2.5 \mathrm{gr} \mathrm{Cl} / 1$ a lo largo de todo el ciclo anual. Durante la época de estudio, las mayores precipitaciones se produjeron en los meses de noviembre de 1982, febrero, noviembre y diciembre de 1983. Como se puede observar en la figura 2, la primera de ellas se manifiesta claramente en la concentración de cloruros de las tres lagunas, la segunda se hace notar en los meses de febrero a marzo, mientras que las dos últimas pensamos que su mayor efecto se produce fuera del ciclo de estudio. El descenso en la concentración de cloruros observado en el mes de agosto en las tres lagunas, no está correlacionado con ninguno de estos periodos de precipitaciones. Hemos de tener en cuenta que los datos de pluviosidad comentados proceden de la estación meteorológica de Las Palmerillas, cercana a las albuferas, y es posible que la disminución del mes de agosto sea debida a las lluvias que se suelen producir en las cumbres de las altas montañas durante el verano, en este caso en la Sierra de Gador, de donde procede el manantial freático que alimenta a las albuferas.

Las tres albuferas se comportan de forma muy semejante respecto a la concentración de fósforo, no pudiéndose distinguir los dos grupos observados en base a su salinidad, ya que todas están sometidas a una gran presión debido a la cantidad de vertidos que reciben de los cultivos que las rodean. La albufera Honda presenta su máxima concentración de fósforo en otoño, con 3 ugr at $/ 1$, presentando un pequeño máximo primaveral de 1 ugr at/1. Por su parte, la albufera Nueva presenta un máximo estival muy marcado, de 15 ugr at/1, quizás debido a que éste muestreo coincidió con un gran aporte de restos procedentes de los cultivos. De todas formas hemos de tener en cuenta que estos valores, al proceder de análisis realizados en aguas sin filtrar, no son exclusivamente de ortofosfatos, sino que también incluyen parte de fósforo orgánico. La albufera litoral presenta un máximo en septiembre de 3 ugr at $/ 1$. El resto de los valores a lo largo del ciclo se sitúan por debajo de 1 ugr at $/ 1$ en las tres lagunas. 

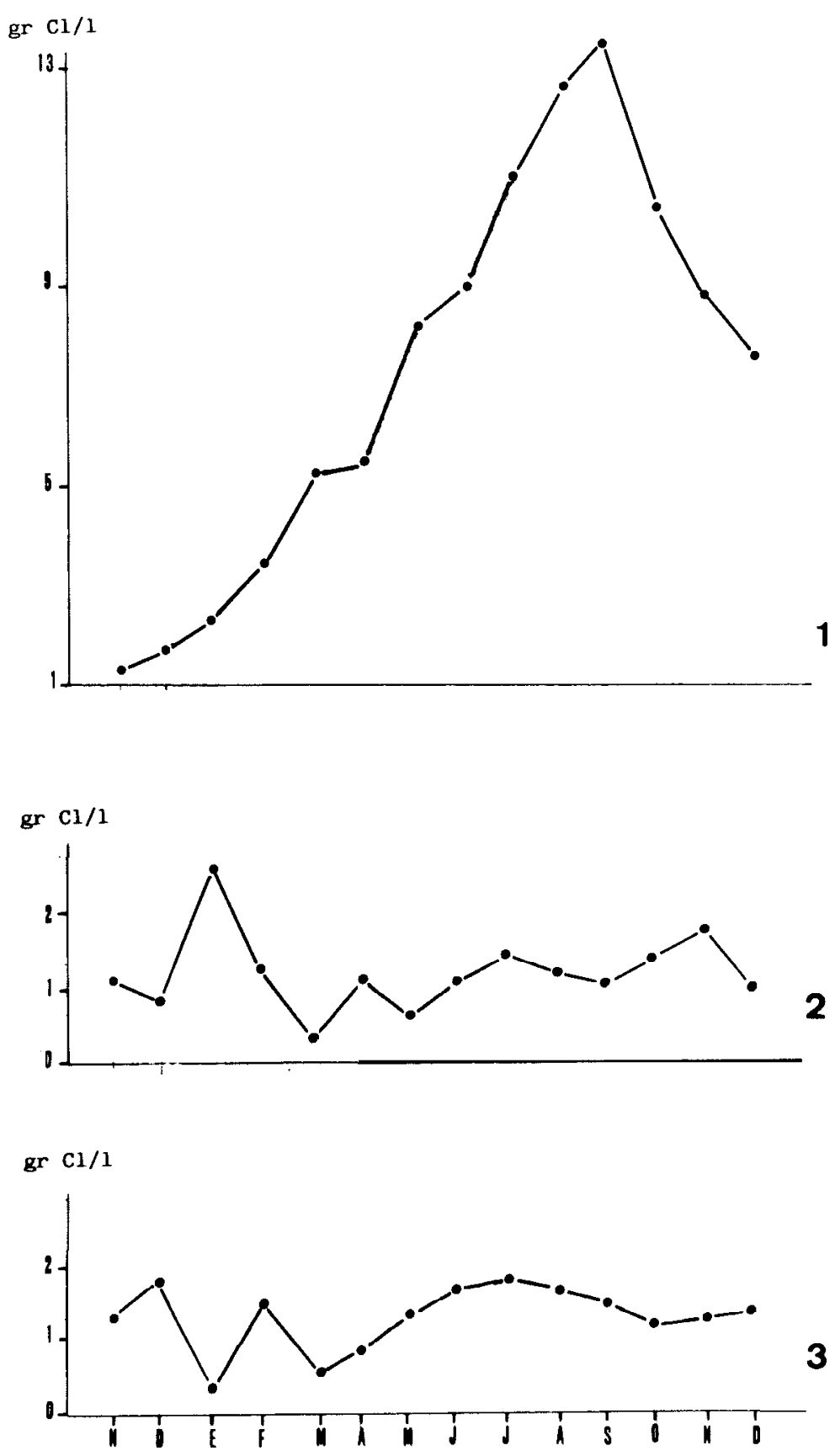

Figura 2.- Ciclo anual de los cloruros en las Albuferas de Adra.

1, Albufera Litoral: 2, Albufera Nueva; 3, Albufera Honda.

Salinity annual cycle in the lagoons of Adra.

1, Albufera Litoral: 2, Albufera Nueva: 3, Albufera Honda 


\section{3) CATALOGO FLORISTICO}

Del total de las 58 especies que aparecen en la tabla l, 49 son planctónicas, inientras que el resto se reparten entre las distintas comunidades bentónicas.

\section{DISCUSION}

De la comparación de los parámetros químicos y la composición especifica de las poblaciones algales de cada una de las lagunas estudiadas, se observa claramente la separación de dos grupos, uno formado por las albuferas interiores: Honda y Nueva, mientras que la albufera Litoral se comporta de forma claramente diferenciada.

La albufera Litoral, con una variación de la salinidad entre 1 y $13 \mathrm{gr} \mathrm{Cl} / 1$, a lo largo del ciclo anual, presenta un fitoplancton claramente diferenciado del de las otras dos lagunas. Entre las cianofíceas destacamos dos especies: Chroococcus turgidus y Gomphosphaeria aponina, ambos taxones es frecuente encontrarlos tanto en ambientes salobres como dulceacuícolas, al localizarlos sólo en esta laguna y no en las dos interiores, donde la salinidad es apreciablemente inferior, consideramos, como ya apuntaron otros autores, que se debe tratar de razas ecológicas más que de especies eurihalinas con un amplio margen de tolerancia. Otras cuatro especies: Mastogloia baltica, Mastogloia braunii, Diploneis didyma y Navicula barberi, las hemos localizado de forma abundante sólo en esta laguna, siendo consideradas como especies adaptadas a medios de elevada salinidad. Otra serie de taxones se encuentran también presentes, además de en la albufera litoral, en las dos interiores, por lo que pueden considerarse como especies que soportan amplios márgenes de salinidad, de hecho algunos taxones tales como: Tetraselmis subcordiformis, Amphora ovalis, Nitzschia apiculata, Cyclotella meneghiniana y Achnanthes hrevipes var. intermedia. son considerados como eurihalinos. Algunos de ellos están muy pobremente representados en las albuferas interiores y sólo están presentes cuando se dan los mayores valores de salinidad, tal es el caso de T. subcordiformis y M. baltica (Tomas, 1982), por lo que pueden ser consideradas como especies mesohalinas que invaden las albuferas interiores en situaciones muy puntuales. Lagunas de salinidad semejante a la albufera Litoral han sido estudiadas por Baudin (1980) y Comin (1984), mostrando una composición específica diferente a la observada en ésta.

Las dos albuferas interiores forman un grupo muy homogéneo en cuanto a su salinidad, reserva alcalina y composición específica. De un total de 36 especies planctónicas localizadas entre las dos lagunas, 16 son comunes a ambas, lo que nos indica que debe existir una gran semejanza entre ellas. De las 16 especies comunes, 8 son frecuentes en ambientes salobres: Coelosphaerium minutissimum, Spirulina major. Chaetoceros sp., Synedra pulchella, Amphiprora paludosa, Amphora coffeaeformis, Nitzschia obtusae y Bacillaria paradoxa; ésta última especie de carácter eurihalino (Carpelan, 1978), por lo que era de esperar que estuviera presente en las tres lagunas. Otra serie de especies, además de alguna de las anteriores, como C. minutissimum a parte de su carácter halobio presentan un claro matiz eutrófico, destacando: Merismopedia punctata, Monoraphidium contortum, Scenedesmus quadricauda, Cocconeis placentula, Gonphonema acuminatum y Nitzschia microcephala.

Existen una serie de especies que sólo hemos localizado en una de las dos albuferas interiores. Respecto a las encontradas sólo en la albufera Nueva, predominan las que podríamos considerar halófilas, observadas también en otros ambientes de marcada mineralización como las formaciones tofícolas (Sánchez \& Gil, 1981), tal es el caso de Rhopalodia gibba; entre las halófilas se encuentran: Epithemia sorex, Cosmarium cf. wembaerense, Nitzschia closterium y Campilodiscus sp. Tanto Budde (1932) como Margalef (1951) incluyen a la mayoría de estas especies en asociaciones consideradas como oligohalinas. La albufera Honda, además de ofrecer una concentración salina levemente inferior, presenta un menor volumen de agua, por lo que el efecto de la contaminación orgánica procedente de los cultivos debe ser mayor. Por ello, en su composición específica particular predominan las especies de carácter eutrófico sobre las salobres, estas especies de matiz marcadamente eutrófico serían: Oscillatoria princeps, Dictyosphaerium pulchellum, Euglena tripteris, Phacus orhicularis y Euglena sp., fundamentalmente. De acuerdo con Carpelan (1964)consideramos que en el interior de la laguna la concentración de nutrientes puede ser más importante que los gradientes de salinidad para explicar la distribución de gran cantidad de especies. Peridinium sp. es la especie que se comporta como dominante en las dos lagunas durante la mayor parte del año, especialmente durante el verano.

Respecto al resto de las comunidades algales, también se diferencian claramente los dos grupos de lagunas delimitados con anterioridad, considerando como parámetro fundamental la salinidad. La albufera Litoral presenta un plocon formado fundamentalmente por Cladophora sp., Rhizoclonium hierogliphicum subsp. riparium, Dichothrix gypsophyla y Croodactylon smaragdina, las dos últimas especies no son exclusivas de aguas salobres, ya que con anterioridad las hemos observado en ambientes tofícolas, en aguas con alto grado de mineralización pero sin cloruros. El plocon de las albuferas interiores se encuentra formado principalmente por Cladophora sp., observando en 
División CYANOPHYTA

Chroococcus dispersus (Kissl.) Lemm.

Chroococcus turgidus (Kutz.) Näg.

Gloeocapsa crepidinum Thuret

Merismopedia punctata Meyen

Merismopedia tenuissima Meyen

Coelosphaerium minutissimum Lemm.

Gomphosphaeria aponina Kutz.

Rhabdoderma sp.

Johannesbaptista pellucida(Dick.) Taylor \& Drouet

Dichothrix gypsophylla(kitz.)Born. \& Flah.

Spirulina major Kutz.

Oscillatoria geminata Menegh.

Oscillatoria princeps Vauch.

Lyngbya confervoides Ag.

Lyngbya halophylla Hansg.

División PYRROPHYTA

Cryptomonas erosa Ehr.

Peridinium sp.

División EUGLENOPHYTA

Euglena tripteris (Duj.)Klebs

Euglena sp.

Phacus orbicularis Hubner

División RHODOPHYTA

Chroodactylon smaragdina Reinsch.

División CHROMOPHYTA

Cyclotella meneghlniana Kütz.

Chaetoceros muelleri Lemm.

Chaetoceros sp.

Synedra pulchella Kutz.

Cocconeis placentula Ehr.

Achnanthes brevipes Ag.

var. intermedia (Riitz.) )Cleve

Mastogloia baltica Grunow

Mastogloia brauni

Pleurosigma elongatum W.Smith

Diploneis didyma Ehr.

Navicula cryptocephala Kutz.

Navicula barbari Hendey

Amphiprora paludosa W.Smith

Amphora coffeaeformis Ag.

Amphora ovalis Kutz.

Gomphonema acuminatum Ehr.

Epithemia sorex Kutz.

Rhopalodia gibba Kutz.

Bacillaria paradoxa Gmel in

Nitzschia apiculata (Greg.) Grun.

Nitzschia closterium (Ehr.)W.Smith

Nitzschia microcephala Grun.

Nitzschia obtusae W.Smith

Campilodiscus sp.

División CHLOROPHYTA

Tetraselmis subcordiformis (wille) Hazen

Monoraphidium contortum (Thur \& Breb.) Leg

Dictyosphaerium pulchellum Wood

Scenedesmus ecornis (Ralfs) Chod.

Scenedesmus quadricauda (Turp.) Breb.

Ulothrix rorida Thuret

Ulothrix subconstricta G.S.West

Cladophora sp.

Rhizoclonium hierogliphicum (Ag.) Kitz

subsp. riparıum (Hervey)Stockmay

Cosmarium $\mathrm{cf}$. wembaerense Schmidle

Closterium sp.

Mougeotia sp.

Spirogyra sp. 
algunas zonas de la albufera Honda masas compuestas por Ulothrix subconstricta, Mougeotia sp. y Spirogyra sp. Comunidades claramente bentónicas (pecton) sólo hemos observado en la albufera litoral, ya que las otras presentan un fondo fundamentalmente arenoso. Estaban formadas por dos especies: Lynghya halophylla y Glococapsa crepidinum.

\section{AGRADECIMIENTOS}

Al Instituto de Estudios Almerienses que financio el estudio limnológico de dos localidades almerienses. del cual se ha extractado el presente trabajo. Al profesor X. Tomas que nos aclaró la identidad de algunas especies de diatomeas.

\section{BIBLIOGRAFIA}

Baudin, J.P. 1980. C'ontribution a I'etude écologique des milieux saumâtres méditerranées. 11 Lepeuplement de I'etang de Cistis. Vie el Milieu, 30(3-4): 303-308.
Budde, H. 1932. Die algenflora westfälicher Salinen und Salzgewasser. Arch.f. Hydrobiologie, 23: 462-490.

Carpelan, L.H. 1964. Effects of salinity on algal distribution. Ecology, 45(1): 70-77.

Carpelan, L.H. 1978. Evolutionary euryhalinity of diatoms in changing environments. Nova Hedwigia, 29: 489-526.

Comin, F.A. 1984. Características físicas y químicas y fitoplancton de las lagunas costeras Encañizada, Tancada y Buda (Delta del Ebro). Oecol aquatica, 7: 79-162.

Golterman, H.L. \& R.S. Clymo. 1969. Methods for chemicals analysis of fresh-nater. I.B.P. Hand-book, n. ${ }^{\circ}$ 8. Ed. Blackwell. Oxford.

Margalef, R. 1951. Materiales para la hidrobiología de la isla de Ibiza. Publ. Inst. Biol. Apl., 8: 9-70.

Murphy, J. \& J.B. Riley. 1962. An modified single method for the determination of phosphate in natural waters. Anal Chim. Acta. 27: 31

Sánchez, P.M. \& J.A. Gil. 1982. Vegetación criptogámica de las tobas de la provincia de Granada. Coll. Bot., 13(1): 231-245.

Tomas, X. 1982. El género Mastogloia en los sistemas acuáticos del litoral mediterráneo español. Coll. Bol., 13(2): 929-944. 